\title{
Using Brief Experimental Analysis to Increase Food Consumption for an Adult with an Eating Disorder: A Case Study
}

\author{
Michael I. Axelrod \\ Human Development Center, \\ University of Wisconsin - Eau Claire, Eau Claire, WI USA.
}

\begin{abstract}
The present study investigated the utility of brief experimental analysis applied to reinforcer assessments in providing a data-based course of treatment targeting food consumption for an adult with Anorexia. Results of two brief experimental analyses indicated the subject consumed more grams of food during the contingent positive attention condition when compared to three other conditions. A brief extended analysis based on the results of the experimental analyses found that positive attention increased the subject's food consumption during lunch and dinner. Results provide preliminary evidence for using brief experimental analyses for identifying contingent relationships between food consumption and maintaining reinforcers. This might be helpful for those developing prescriptive treatments to increase food consumption of adults with Anorexia.
\end{abstract}

Keywords: Anorexia Nervosa; treatment; brief experimental analysis; reinforcer assessment

\section{INTRODUCTION}

Anorexia Nervosa (AN) is characterized by extreme patterns of restricted eating resulting in significant weight loss, fear of gaining weight, and body image disturbances (APA, 2013). AN is considered the most severe eating disorder, as it has high morbidity and mortality rates, is chronic, and requires intensive and ongoing intervention (Resmark, Herpertz, HerpertzDahlmann, \& Zeeck, 2019). Unfortunately, research on the treatment of AN is mixed. For example, a systematic review of randomized controlled studies of medication therapy, Cognitive-behavior therapy (CBT), and medication plus CBT concluded the evidence for these commonly used treatment was weak (Bulik, Berkman, Brownley, Sedway, \& Lohr, 2007). More recently, a meta-analysis of randomized and nonrandomized controlled studies offered more optimistic findings (Zeeck et al., 2018). Specifically, the researchers identified several therapeutic models, including CBT and family-based treatment, that demonstrated strong positive effects. However, methodological limitations including a lack of untreated comparison groups plague the literature and many experts on AN have called for more research on treatment approaches (e.g., Resmark et al., 2019).

Evidence-based treatment models of AN generally begin with weight gain and weight management through meal planning, self-monitoring, and psychoeducation (Resmark et al., 2019; Stefano, Bennett, Bozsik, Hudson, \& Whisenhunt, 2018). These intervention features attempt to address caloric restriction, although cognitive features of AN (e.g., distorted body image) and specific motivational variables (e.g., not believing the problem is serious) are likely to interfere with progress and overall outcomes (Fitzsimmons-Craft et al., 2019). Furthermore, problems associated comorbid psychiatric conditions, such as anxiety and depression, might make treating AN even more challenging (Shilton et al., 2019). Finally, Kazdin, FitzsimmonsCraft, and Wilfley (2017) indicated a significant research-practice gap in providing treatment to individuals with AN. Specifically, these researchers noted evidence-based treatments 
identified through controlled studies rarely extend to practice settings. They highlighted key characteristics of treatment delivery models that could reach more individuals with AN including employing new intervention approaches that could be modified to meet setting specific circumstances.

Given the aforementioned issues, identifying novel approaches to treating AN might be necessary to supplement current intervention practices and expand treatment options. Perhaps one avenue to guide treatment is through the use of experimental analysis. Experimental analysis is a behavioral assessment method that emphasizes relationships between an individual's behavior and specific environmental events. A distinctive feature of experimental analysis is that observations occur "within tightly controlled, single-case designs to demonstrate that environmental variables affect or change the responding of target behavior (Wacker, Berg, Harding, \& Cooper-Brown, 2011, p. 165). Said differently, the process of experimental analysis identifies specific environmental conditions which cause a change in an observed behavior. Brief experimental analysis has been applied to the assessment of behavioral function, interventions, and reinforcers (Axelrod, 2017). For reinforcer assessments, the procedure involves systematically introducing different reinforcers (e.g., positive attention, access to tangible items, escape from aversive stimuli) and observing the target behavior. Noteworthy changes in the target behavior following the introduction of a specific reinforcer indicates a likely contingent relationship between the behavior and reinforcer. Brief experimental analysis involving reinforcer assessment has not yet been applied to caloric restriction and food intake. The current study attempted to identify reinforcers maintaining food refusal through the use of two brief experimental analyses and evaluate the findings via an extended analysis.

\section{Participants and Setting}

\section{METHOD}

Anne was a 19-year-old Caucasian female residing in an acute care psychiatric hospital. She was voluntarily admitted after making multiple self-harm statements. She had a previous diagnosis of AN, which was confirmed by a psychiatric nurse practitioner during the hospital's intake assessment. Anne was referred for a psychological consultation one week after being admitted because she was refusing to eat food during mealtime. According to her medical record, Anne had lost 10 pounds in the week since her admission. Her history included previous episodes of food refusal in other psychiatric placements. Anne's treatment at the hospital included daily individual and group psychotherapy and daily progress meetings with medical staff. Anne's food refusal was not generally discussed during those sessions nor was it discussed on the hospital unit with Anne by hospital staff. Furthermore, Anne did not make comments to staff about her food refusal.

All sessions were conducted in the hospital cafeteria during lunch and dinner. Hospital staff and patients were present in the cafeteria. Anne sat at a table with one designated staff member during mealtime. Meals were made up in the kitchen by a registered dietician and brought to Anne by a cafeteria employee. After the meal, leftover food on the plate was taken to the dietician's office for data collection. Mealtime averaged 21 minutes (range, 15 minutes to 31 minutes). Anne was not aware she was being observed during mealtime.

\section{Dependent Variable}

The dependent variable was percentage of grams of food consumed during each meal. A registered dietician weighed all meals before and after mealtime using a digital food scale. The average grams of food presented for lunch and dinner was 492 and 525, respectively. The dependent variable was calculated by first subtracting the number of grams left on the plate by 
the total number of grams in the meal. The percentage of grams consumed was determined by dividing the grams consumed by the total number of grams in the meal and multiplying by $100 \%$.

\section{Procedure and Experimental Design}

Two brief experimental analyses were conducted for lunch and dinner. Three positive reinforcement conditions (i.e., positive attention, negative attention, access to tangible item) and baseline were implemented across mealtimes. During the baseline condition, the designated staff member provided no attention to Anne during the meal. During the positive attention condition the staff member provided 30-sec of attention (e.g., encouraging statements, praise, small talk) contingent on Anne taking a bite of food or attempting to eat (e.g., picking up eating utensil, placing food on fork). In the negative attention condition, the staff member provided attention (e.g., threats of response cost consequences and meetings with the medical director) contingent on the absence of Anne taking a bite or attempting to take a bite. In the tangible condition, Anne received access to her MP3 player for 1-hr contingent on eating four forkfuls or spoonfuls of food during the meal. Following the brief experimental analyses, a multiple-baseline design across settings was used to evaluate the effects of the positive attention condition on Anne's food consumption. The positive attention condition was selected based on the results of the two brief experimental analyses.

\section{Interobserver Agreement and Procedural Integrity}

Interobserver agreement (IOA) was assessed during 15\% of the meals. A second registered dietician weighed all meals before and after mealtime using the same standard scale. IOA was calculated by dividing one dietician's measurements by the other dietician's measurements and multiplying by $100 \%$. IOA means for the number of grams pre- and post-mean was $99 \%$ (range, 97\% to 100\%) and number of grams left on the plate was $99 \%$ (range, 98\% to 100\%). Procedural integrity was assessed for $50 \%$ of the sessions. Sitting at another table, a trained psychology undergraduate student observed interactions between Anne and the designated staff member using a list of all procedural components. Integrity was observed to be $100 \%$.

\section{RESULTS}

Figure 1 displays the results of the brief experimental analysis and extended analysis for both lunch and dinner. Results from the brief experimental analyses showed that Anne consumed a higher percentage of grams of food during the positive attention condition when compared to the other three conditions. The percentages of grams consumed for the baseline, negative attention, and access to tangible item conditions were all close to zero for both meals. The average percentage of grams consumed during the positive attention condition was approximately $36 \%$ for lunch and $33 \%$ for dinner. The extended analysis found that the positive attention condition increased Anne's food consumption during both lunch and dinner. The mean percentage of grams of food consumed during the baseline conditions was 3.19\% for lunch and $1.30 \%$ for dinner. In contrast, the mean percentage of grams of food consumed during the positive attention conditions was $51.58 \%$ for lunch and $34.80 \%$ for dinner. Overall, Anne consumed a higher percentage of grams of food when given positive attention by staff contingent on Anne's eating or attempts at eating compared to baseline levels. 


\section{Figure 1}

Percentage of grams consumed by Anne per meal across conditions during lunch and dinner.

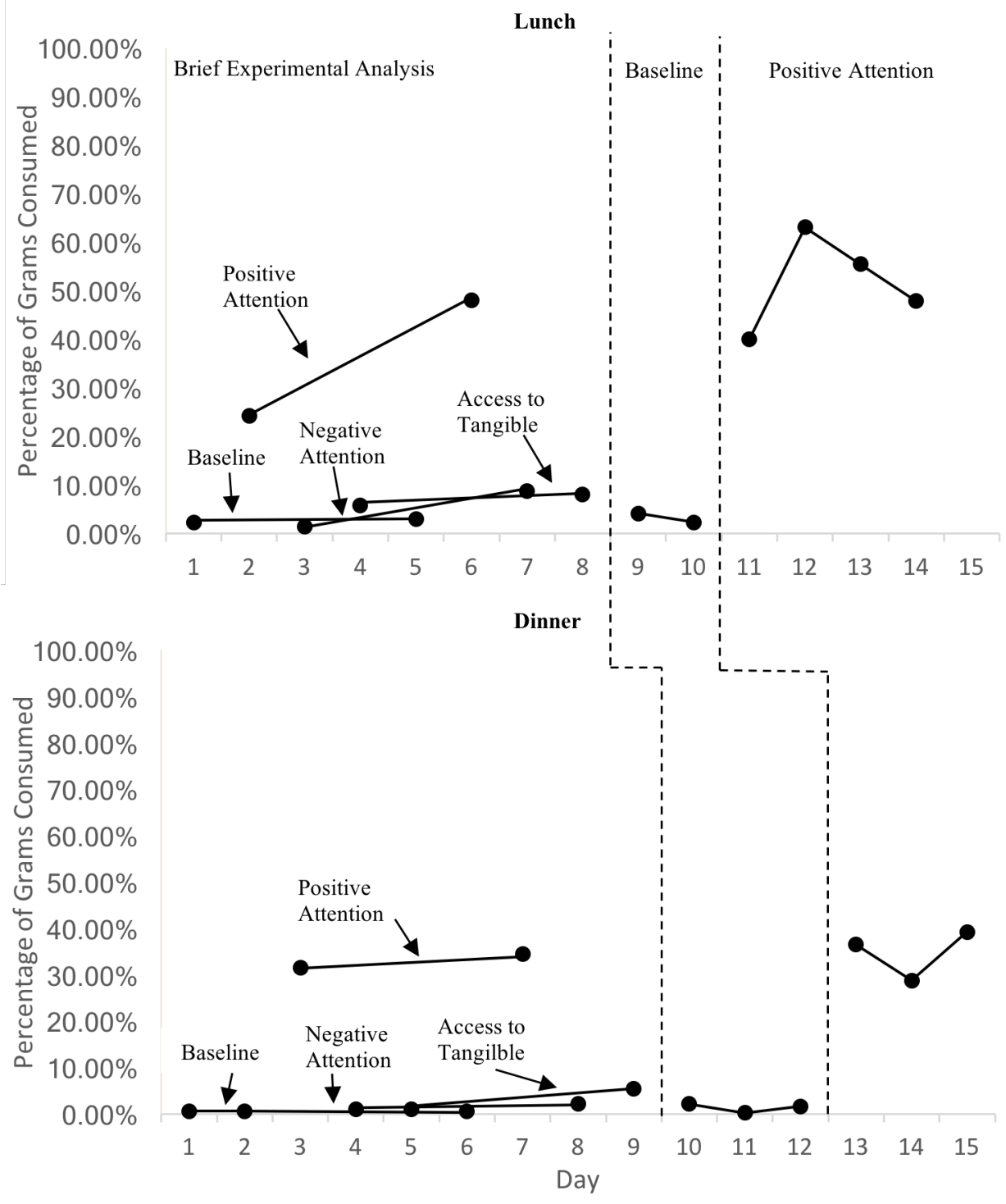

\section{DISCUSSION}

This is the first published case study to employ a brief experimental analysis to identify reinforcers that could increase food consumption for an adult with AN. Results from the study indicated that a brief experimental analysis applied to a reinforcer assessment could empirically select contingent relationships between food consumption and reinforcement. Research on the use of experimental and brief experimental analysis has provided guidance regarding food refusal in pediatric and developmentally delayed populations but has not been applied to adult eating disorders (see Piazza \& Addison, 2007; Piazza, Paten, Gulotta, Sevin, \& Layer, 2003). The present study provides preliminary evidence for using experimental analysis to identify a reinforcer that increase food consumption. Food refusal and Anorexia Nervosa pose significant problems in psychiatric settings (Lock \& Gowers, 2005). Identifying contingent relationships between the behavior of food refusal and its reinforcing consequences using brief experimental analysis might offer a new approach to the treatment of AN. Practitioners treating AN and adult food refusal should consider utilizing brief experimental analyses to select reinforcers that increase food consumption when developing treatment plans.

The case study's findings should be considered exploratory given this was a preliminary investigation with some limitations. For example, the case study represented one subject, 
which prohibits generalization of the study's findings. Future research should attempt to replicate the current study using a larger subject pool and subjects from varying populations in different settings (e.g., community, hospital). Second, concomitant variables might have influenced the results. For example, Anne might have consumed food while on the hospital's unit. While no formal measures were in place, hospital staff reported that Anne did not eat breakfast or snack on the unit. Furthermore, while all attempts were made to control for other treatments (e.g., little to no discussion of food refusal during psychotherapy), it is impossible to know whether other on-going interventions might have contributed to the findings. Future research should attempt to control for extraneous variables associated with treatment as appropriate for the setting. Finally, the length of the treatment phase makes it impossible to conclude the intervention had more than a short-term impact on Anne's food consumption. Future studies should attempt to employ longer treatment phases following the brief experimental analysis so that the utility of the procedure in empirically identifying reinforcers can be established. Despite these limitations, the current case study indicates that a brief experimental analysis could be a promising reinforcer assessment technique in the development of effective treatments for $\mathrm{AN}$ and food refusal and deserves further investigation.

\section{References}

American Psychiatric Association. (2013). Diagnostic and Statistical Manual of Mental Disorders (5 ${ }^{\text {th }}$ edition). Washington D.C.: Author.

Axelrod, M.I. (2017). Behavior analysis for school psychologists. New York: Routledge.

Bulik, C.M., Berkman, N.D., Brownley, K.A., Sedway, J.A., \& Johr, K.N. (2007). Anorexia nervosa treatment: A systematic review of randomized controlled trials. International Journal of Eating Disorders, 40, 310-320.

Fitzsimmons-Craft, E.E., Eichen, D.M., Monterubio, G.E., Firebaugh, L., Goel, N.J., Barr Taylor, C., \& Wilfley, D.E. (2019). Longer-term follow-up of college students screening positive for anorexia nervosa: Psychopathology, help seeking, and barriers to treatment. Eating Disorders. doi: 10.1080/10640266.2019.1610628

Kazdin, A.E., Fitzsimmons-Craft, E.E., \& Wilfley, D.E. (2017). Addressing critical gaps in the treatment of eating disorders. International Journal of Eating Disorders, 50, 170-189.

Lock, J. \& Gowers, S. (2005). Effective interventions for adolescents with anorexia nervosa. Journal of Mental Health, 14, 599-610.

Piazza, C. C. \& Addison, L. R. (2007). Function-based assessment and treatment of pediatric feeding disorders. In P. Sturmey (Ed.), Functional analysis in clinical treatment (pp.129-149). Academic Press: Burlington, MA.

Piazza, C. C., Paten, M. R., Gulotta, C. S., Sevin, B. M., \& Layer, S. A. (2003). On the relative contributions of positive reinforcement and escape extinction in the treatment of food refusal. Journal of Applied Behavior Analysis, 36, 309324.

Resmark, G., Herpertz, S., Herpertz-Dahlmann, B., \& Zeeck, A. (2019). Treatment of anorexia nervosa - new evidence-based guidelines. Journal of Clinical Medicine,8, 1-16. doi: 10.3390/jcm8020153

Shilton, T., Enoch-Levy, A., Giron, Y., Yaroslavsky, A., Amaiz, R., Gothelf, D.,...Stein, D. (2019). A retrospective case series of electroconvulsive therapy in the management of comorbid depression and anorexia nervosa. International Journal of Eating Disorders. Advance online publication. doi: 10.1002/eat.23181

Stefano, E.C., Bennett, B.L., Bozsik, F., Hudson, D.L., \& Whisenhunt, B. L. (2018). Eating. In S. Hupp (Ed.), Child and Adolescent Psychotherapy: Components of Evidence-Based Treatments for Youth and their Parents (pp. 224-245). New York: Cambridge University Press.

Wacker, D.P., Berg, W.K., Harding, J.W., \& Cooper-Brown, L.J. (2011). Functional and structural approaches to behavioral assessment. In W.W. Fischer, C.C. Piazza, \& Roane, H.S. (Eds.), Handbook of Applied Behavior Analysis (pp. 165-181). New York: Guilford.

Zeeck, A., Herpertz-Dahlmann, B., Friederich, H., Brockmeyer, T., Resmark, G., Hagenah, U.,...Hartmann, A. (2018). Psychotherapeutic treatments for anorexia nervosa: A systematic review and network meta-analysis. Frontiers in Psychiatry, 9, 1-14. doi: 10.3389/fpsyt.2018.00158 